\title{
Desafios da implementação do BIM em microempresas da indústria AEC
}

\author{
Challenges of BIM implementation in micro-sized firms from AEC \\ Desafíos de la implementación del BIM en microempresas de la industria AEC
}

Recebido: 14/01/2022 | Revisado: 18/01/2022 | Aceito: 23/01/2022 | Publicado: 25/01/2022

\author{
Marllon Christian José Martins \\ ORCID: https://orcid.org/0000-0001-9619-2075 \\ Universidade Federal de São João del-Rei, Brasil \\ E-mail: marllon.cjm@gmail.com \\ Natalia Assunção Brasil Silva \\ ORCID: https://orcid.org/0000-0002-4296-6407 \\ Universidade Federal de São João del-Rei, Brasil \\ E-mail: natalia.brasil@ufsj.edu.br \\ Emmanuel Kennedy da Costa Teixeira \\ ORCID: https://orcid.org/0000-0001-7598-0240 \\ Universidade Federal de São João del-Rei, Brasil \\ E-mail: emmanuel.teixeira@ufsj.edu.br
}

\begin{abstract}
Resumo
Este trabalho visa determinar os desafios no processo de implementação do Building Information Modeling (BIM) nas microempresas do setor de Arquitetura, Engenharia e Construção (AEC). A metodologia aplicada consistiu em uma abordagem exploratória, a partir do levantamento de opiniões dos profissionais atuantes no setor de AEC, acerca da aplicabilidade e nível de dificuldade dos desafios no processo de implementação do BIM à realidade das microempresas. Para levantamento de opiniões valeu-se da aplicação de um questionário on-line elaborado em conformidade com a revisão da literatura técnico-científica sobre o assunto. No estudo foi possível identificar e avaliar o nível de dificuldade de 41 desafios no processo de implementação do BIM à realidade das microempresas, evidenciando que o desconhecimento, por parte dos clientes de empresas, sobre as vantagens do BIM é o mais crítico entre eles. Foram identificados 13 desafios não citados na literatura existente. Desse modo, ao organizar, avaliar e categorizar os desafios, conclui-se que o desenvolvimento de um plano de implementação, com estratégias baseadas nos desafios identificados nesta pesquisa, pode auxiliar no processo de implementação do BIM nas microempresas do setor de AEC.
\end{abstract}

Palavras-chave: BIM; Implementação; Desafios; Arquitetura, Engenharia e Construção.

\begin{abstract}
This work aims to determine the challenges in the process of implementing Building Information Modeling (BIM) in micro-enterprises in the Architecture, Engineering and Construction (AEC) sector. The methodology applied consisted of an exploratory approach, based on the survey of opinions of professionals working in the AEC sector, about the applicability and level of difficulty of the challenges in the process of implementing BIM to the reality of micro-enterprises. To survey opinions, it was used an online questionnaire prepared in accordance with the review of the technical-scientific literature on the subject. In the study, it was possible to identify and evaluate the level of difficulty of 41 challenges in the process of implementing BIM to the reality of micro-enterprises, showing that the lack of knowledge, on the part of business customers, about the advantages of BIM is the most critical among them. 13 challenges not mentioned in the existing literature were identified. Thus, by organizing, evaluating and categorizing the challenges, it is concluded that the development of an implementation plan, with strategies based on the challenges identified in this research, can help in the process of implementing BIM in micro-enterprises in the AEC sector.
\end{abstract}

Keywords: BIM; Implementation; Challenges; Architecture, Engineering and Construction.

\section{Resumen}

Este trabajo tiene como objetivo determinar los desafíos en el proceso de implementación de Building Information Modeling (BIM) en microempresas del sector de Arquitectura, Ingeniería y Construcción (AEC). La metodología aplicada consistió en un enfoque exploratorio, basado en la encuesta de opiniones de profesionales que trabajan en el sector AEC, sobre la aplicabilidad y el nivel de dificultad de los desafíos en el proceso de implementación de BIM a la realidad de las microempresas. Para relevamiento de opiniones se utilizó un cuestionario en línea elaborado de acuerdo con la revisión de la literatura técnico-científica sobre el tema. En el estudio, fue posible identificar y evaluar el nivel de dificultad de 41 desafíos en el proceso de implementación de BIM a la realidad de las microempresas, mostrando que la falta de conocimiento, por parte de los clientes comerciales, sobre las ventajas de BIM es el más crítico entre ellos. Se identificaron 13 desafíos no mencionados en la literatura existente. Así, al organizar, evaluar y 
categorizar los desafíos, se concluye que el desarrollo de un plan de implementación, con estrategias basadas en los desafíos identificados en esta investigación, puede ayudar en el proceso de implementación de BIM en las microempresas del sector AEC.

Palabras clave: BIM; Implementación; Desafíos; Arquitectura, Ingeniería y Construcción.

\section{Introdução}

A Modelagem da Informação da Construção, conhecida por BIM (Building Information Modelling), tem sido implementada com sucesso no cenário mundial (Smith, 2014). Seu uso tem proporcionado mudanças na forma tradicional de gerenciar e projetar da indústria da Arquitetura, Engenharia e Construção (AEC), alterando os processos e padrões consolidados no setor (Gomes et al., 2018).

O BIM é definido como "uma tecnologia de modelagem e um conjunto associado de processos para produzir, comunicar e analisar modelos de construção" (Eastman et al., 2021, p.21), que também pode ser caracterizado como um recurso tecnológico que permite a atribuição de informações à modelagem, incentiva o trabalho colaborativo e facilita a tomada de decisões (Campestrini et al., 2015).

$\mathrm{Na}$ literatura técnico-científica são elencadas diversas vantagens da aplicação do BIM em relação à metodologia tradicional do Computer-Aided Design (CAD), principalmente, na elaboração de projetos. Dentre as vantagens, tem-se a melhora da produtividade, pois o BIM facilita as revisões de projeto, torna a extração de quantitativos mais rápida e precisa, possibilita a melhor compatibilização e verificação de interferências entre as disciplinas de arquitetura, estrutura, instalações, dentre outras (Costa et al., 2015; Santos et al., 2016; Succar et al., 2016; Steiner, 2016; Santos, 2016; ABDI, 2017; Maína, 2018; Carvalho, 2019; Coutinho et al., 2021; Eastman et al., 2021). Além disso, o BIM proporciona significativas melhorias nas simulações computacionais dos empreendimentos, o que privilegia a transparência nos serviços de orçamentação e licitação, além de melhorar a gestão de projetos e das obras (Governo do Paraná, 2018).

Diante dos benefícios e a crescente utilização do BIM, o governo brasileiro promulgou textos legais infraconstitucionais, na forma de lei e decretos, para promover estratégias de disseminação do BIM (intitulada Estratégia BIM BR) e proporcionar, de forma gradual, um ambiente adequado ao investimento na área e a sua difusão no país (Brasil, 2018; Castro, 2020). Dentre os textos infraconstitucionais diretamente relacionados ao BIM, destaca-se o Decreto n. 9.377 (Brasil, 2017), Decreto n. 9.983 (Brasil, 2019), Decreto n. 10.306 (Brasil, 2020), a Lei n. ${ }^{\circ} 14.133$ (Brasil, 2021).

Observa-se que a adoção do BIM está planejada para ocorrer de forma gradual no setor público e, conforme CBIC (2016a), o processo de implementação no setor privado também deverá ser gradual. Isso evidencia que, para ter sucesso nesse processo e, assim, usufruir dos benefícios do BIM em sua plenitude, deve ser estabelecido, por parte das empresas, um planejamento estratégico de longo prazo, com objetivos, etapas e prazos adequadamente definidos e estruturados.

Nesse sentido, a existência de estudos e referências podem ser de grande auxílio para o planejamento da implementação. Entretanto, como apresentado no mapeamento realizado por Checcucci (2019) sobre publicações realizadas de 2013 a 2018 referentes ao BIM, há uma escassez de trabalhos científicos sobre a sua implementação em empresas brasileiras, visto que dos 143 trabalhos publicados neste período, a maioria concentrou-se em aspectos de projeto. Dentre os publicados, apenas 12 trataram especificamente da implementação do BIM, o que, comparativamente, demonstra a necessidade de mais estudos a respeito desse assunto.

Aspectos como a escassez de estudo sobre a implementação do BIM e este ser um instrumento inovador no contexto da indústria AEC brasileira (Cardoso, 2020), podem gerar diversos desafios para as empresas que desejam implementar o BIM (Checcucci, 2019). Este fato, aliado aos riscos e incertezas intrínsecos à decisão de inovar (Silva \& Dacorso, 2014), podem desencorajar a implementação do BIM, visto que tomar decisões estratégicas frente a um cenário pouco conhecido pode ser uma tarefa desafiadora para os gestores dessas empresas. Mas, como destacado por Carvalho (2015, p. 167), “ao inovar em 
direções pouco exploradas pelos concorrentes, as empresas podem alcançar uma oferta diferenciada que pode tornar-se fonte de vantagem competitiva".

Conforme o mapeamento apresentado pelo DataSebrae (2020), o Brasil possuía, no ano de realização do censo, 78.691 empresas de serviços de engenharia e 21.201 de serviços de arquitetura. Dentre essas empresas, aquelas com até nove colaboradores, isto é, as microempresas (Sebrae, 2020), mostram-se muito significativas para o setor, por consistirem em 66\% do total de empresas dessa categoria (DataSebrae, 2020). Diante da importância das microempresas na indústria AEC brasileira, torna-se notório a relevância em efetuar uma análise da implementação do BIM nas empresas desse porte.

Logo, para auxiliar no processo de implementação do BIM nas microempresas do setor AEC, o presente estudo visa determinar os desafios existentes no processo de implementação do BIM nas microempresas da indústria AEC.

\section{Metodologia}

Para determinar os desafios presentes no processo de implementação do BIM nas microempresas da indústria AEC o presente estudo foi dividido em quatro etapas: i) Elaboração do questionário; ii) Aplicação do questionário; iii) Tratamento dos dados coletados; e iv) Análise das informações.

$\mathrm{Na}$ primeira etapa "Elaboração do questionário", houve uma pesquisa exploratória, baseada nos procedimentos descritos por Lakatos e Marconi (2017). Esta etapa consistiu na revisão bibliográfica em fontes acadêmicas através de descritores específicos (palavras-chave) em mecanismos de busca on-line, que resultaram na obtenção de guias práticos, mapeamentos, levantamentos estatísticos, trabalhos acadêmicos e artigos de periódicos relacionados ao tema delimitado. A partir do resultado dessa pesquisa, foram obtidas informações para fundamentação e elaboração das questões necessárias ao cumprimento do objetivo do trabalho.

$\mathrm{Na}$ etapa de "Elaboração do questionário", para identificar os diversos desafios atrelados à implementação do BIM nas empresas, considerou-se neste estudo, diversos autores nacionais e internacionais, dentre eles Migilinskas et al. (2013), Stehling \& Arantes (2014), Dantas Filho et al. (2015), Liu (2015), CBIC (2016b), Pereira (2016), Coelho (2017), Ghaffarianhoseini (2017), Sun et al. (2017), Gomes et al. (2018), CBIC (2018), Zeglin (2018), Chan et al. (2019), Landim (2020), Sienge e Grant Thorton (2020) e Eastman et al. (2021).

Ao analisar as pesquisas dos autores supracitados, foi possível identificar que alguns desafios se mostram semelhantes ou complementares, tornando possível elencar 42 desafios distintos. E para obter uma melhor organização e clareza dos desafios é interessante, conforme feito por Sun et al. (2017) e Liu (2015), distribuir e organizar os desafios da implementação do BIM nas empresas em categorias. Baseado na categorização realizada pelos autores supracitados foi possível determinar cinco categorias principais, sendo elas: i) Recursos humanos, clientes e processos; ii) Informação e tecnologia; iii) Recursos e investimentos; iv) Gerenciamento e referências; e v) Aspectos legais. Conforme as categorias definidas, são apresentadas na Tabela 1 os desafios atrelados à implementação do BIM nas empresas. 
Tabela 1. Desafios relacionados à implementação do BIM nas empresas.

\begin{tabular}{|c|c|c|}
\hline Categoria & Código & Desafio \\
\hline \multirow{8}{*}{ 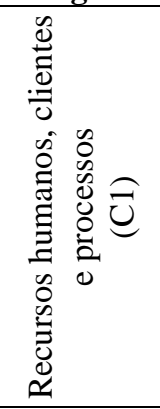 } & C1.1 & Carência de profissionais suficientemente capacitados em BIM. \\
\hline & $\mathrm{C} 1.2$ & Desconhecimento, por parte dos clientes, sobre as vantagens do BIM. \\
\hline & $\mathrm{C} 1.3$ & Desconhecimento, por parte dos profissionais, sobre as vantagens do BIM. \\
\hline & $\mathrm{C} 1.4$ & Dificuldade quanto à natureza individualizada no processo de projeto e construção (não é integrado). \\
\hline & $\mathrm{C} 1.5$ & Falta de interesse pelo trabalho colaborativo e em equipe, aspectos essenciais à metodologia BIM. \\
\hline & C1.6 & $\begin{array}{l}\text { Inércia e resistência, por parte dos profissionais e organizações, em mudar a forma de trabalho e aceitar } \\
\text { inovações. }\end{array}$ \\
\hline & $\mathrm{C} 1.7$ & Necessidade de reestruturação do processo de projeto. \\
\hline & $\mathrm{C} 1.8$ & $\begin{array}{l}\text { Profissionais visualizam o BIM como uma ameaça a seu posto de trabalho e receiam o aumento de } \\
\text { demandas e atribuições ao migrar para o BIM. }\end{array}$ \\
\hline \multirow{10}{*}{ 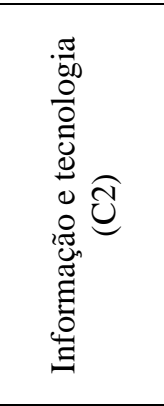 } & $\mathrm{C} 2.1$ & Deficiência no ensino técnico e universitário brasileiro quanto ao ensino do BIM. \\
\hline & $\mathrm{C} 2.2$ & Dificuldade na aprendizagem e uso devido à complexidade da tecnologia. \\
\hline & $\mathrm{C} 2.3$ & Dificuldade na escolha dos softwares e hardwares (equipamentos) apropriados as demandas da empresa. \\
\hline & $\mathrm{C} 2.4$ & Falta de biblioteca, informações e parâmetros dos produtos necessários à modelagem em BIM. \\
\hline & $\mathrm{C} 2.5$ & Falta de demanda ou exigência (por parte dos clientes) para projetos detalhados em BIM. \\
\hline & $\mathrm{C} 2.6$ & Falta de treinamento e suporte técnico em português para as ferramentas. \\
\hline & $\mathrm{C} 2.7$ & Falta de um software único para todas as disciplinas de projeto. \\
\hline & $\mathrm{C} 2.8$ & Normas técnicas do BIM ainda não estão definidas ou amplamente adotada. \\
\hline & $\mathrm{C} 2.9$ & Perda de informações e parâmetros no processo de comunicação entre softwares BIM. \\
\hline & $\mathrm{C} 2.10$ & Problemas de interoperabilidade (trabalho em conjunto e comunicação) entre softwares. \\
\hline \multirow{10}{*}{ 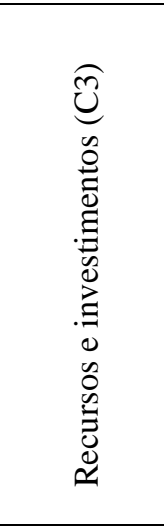 } & C3.1 & Baixo ou nenhum retorno do investimento realizado para implementação no curto prazo. \\
\hline & C3.2 & Custo com treinamentos, capacitação e mudança dos processos internos. \\
\hline & C3.3 & $\begin{array}{l}\text { Custo do investimento em infraestrutura de TI (servidores, rede, banda de Internet, serviços de } \\
\text { armazenamento em nuvem, etc.). }\end{array}$ \\
\hline & C3.4 & $\begin{array}{l}\text { Custo do investimento na aquisição e melhorias de infraestrutura tecnológica (hardwares, softwares, } \\
\text { licenças e bibliotecas BIM). }\end{array}$ \\
\hline & C3.5 & Dificuldade em precificar projetos elaborados em BIM. \\
\hline & C3.6 & $\begin{array}{l}\text { Hesitação quanto ao investimento na implementação do BIM, devido ao cenário de crise e incertezas na } \\
\text { economia brasileira. }\end{array}$ \\
\hline & $\mathrm{C} 3.7$ & Problemas e riscos devido à redução de produtividade ocasionados pela curva de aprendizagem. \\
\hline & C3.8 & Problemas quanto à competitividade da empresa no mercado tendo poucos recursos. \\
\hline & C3.9 & Quadro de funcionários insuficiente para a implementação e/ou utilização do BIM. \\
\hline & C3.10 & Tempo demandado para implementação BIM é muito elevado. \\
\hline \multirow{10}{*}{ 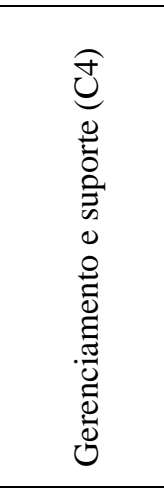 } & C4.1 & $\begin{array}{l}\text { Conflito de comunicação, cooperação e coordenação entre as disciplinas de projeto (arquitetura, } \\
\text { engenharia, complementos, etc.). }\end{array}$ \\
\hline & $\mathrm{C} 4.2$ & Dificuldade em balancear os esforços entre a implementação e os ganhos gerenciais e monetários. \\
\hline & $\mathrm{C} 4.3$ & Dificuldade em conciliar a implementação com as demandas já existentes. \\
\hline & C4.4 & Dificuldade no cálculo e comprovação do retorno sobre o investimento (ROI) no BIM. \\
\hline & $\mathrm{C} 4.5$ & Dificuldade no gerenciamento da mudança cultural. \\
\hline & $\mathrm{C} 4.6$ & Dificuldade no planejamento da implementação do BIM. \\
\hline & $\mathrm{C} 4.7$ & Falta de clareza sobre os papéis de cada membro da equipe durante a implementação. \\
\hline & $\mathrm{C} 4.8$ & Falta de diretrizes ou incentivos por parte dos governos. \\
\hline & C4.9 & Falta de normas técnicas, referências e regulamentos de apoio para implementação do BIM. \\
\hline & C4.10 & $\begin{array}{l}\text { Falta de suporte, orientação ou apoio por parte de superiores ao processo de implementação da } \\
\text { metodologia BIM. }\end{array}$ \\
\hline \multirow{4}{*}{ 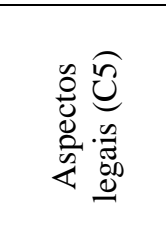 } & C5.1 & Mudanças no escopo dos contratos e licitações em relação à metodologia tradicional CAD. \\
\hline & C5.2 & $\begin{array}{l}\text { Necessidade de cláusulas contratuais para a boa colaboração entre as fases do projeto (sem retenção de } \\
\text { informações importantes). }\end{array}$ \\
\hline & C5.3 & $\begin{array}{l}\text { Problemas quanto aos direitos autorais, acesso, uso e compartilhamento dos múltiplos conjuntos de dados } \\
\text { de projeto. }\end{array}$ \\
\hline & C5.4 & Softwares em desconformidade com as normas brasileiras. \\
\hline
\end{tabular}

Fonte: Autores (2021).

Em conformidade com essa etapa, neste estudo foram formuladas questões direcionadas ao contexto da implementação do BIM em microempresas da indústria AEC. Desse modo, o questionário formado com 12 questões foi dividido em duas seções, conforme descrito na Tabela 2. 
Tabela 2. Objetivo das questões presentes no questionário on-line.

\begin{tabular}{|c|c|}
\hline Seções & Objetivos \\
\hline \multirow{3}{*}{$\begin{array}{l}\text { Informações } \\
\text { gerais (seis } \\
\text { questões) }\end{array}$} & Verificar o conhecimento da definição de BIM, para validação das participações na pesquisa. \\
\hline & $\begin{array}{l}\text { Coletar de informações sobre o participante (faixa etária) e empresa (porte, setor de atuação e localização) } \\
\text { em que possui vínculo. }\end{array}$ \\
\hline & Verificar a expertise e intenção do participante/empresa quanto a implementação do BIM. \\
\hline \multirow{5}{*}{$\begin{array}{l}\text { Desafios na } \\
\text { implementação } \\
\text { do BIM em } \\
\text { microempresas } \\
\text { (seis questões) }\end{array}$} & $\begin{array}{l}\text { Verificar o conhecimento dos participantes, quanto a existência de instrumento facilitador ou normas } \\
\text { técnicas relativas à implementação do BIM. }\end{array}$ \\
\hline & $\begin{array}{l}\text { Validação de } 42 \text { desafios }{ }^{(2)} \text { identificados na literatura quanto a sua aplicabilidade no contexto da } \\
\text { implementação do BIM em uma microempresa e verificar o nível de dificuldade gerado por eles neste } \\
\text { processo sob a ótica do participante. Para tal, valeu-se das opções "não se aplica", "alto", "médio" e } \\
\text { "baixo". }\end{array}$ \\
\hline & $\begin{array}{l}\text { Verificar a ordem de relevância das categorias no processo de implementação do BIM nas } \\
\text { microempresas; }\end{array}$ \\
\hline & Coletar desafios adicionais que não foram citados na literatura. \\
\hline & $\begin{array}{l}\text { Coletar opiniões sobre iniciativas e políticas governamentais que poderiam contribuir para minimizar, } \\
\text { impedir e/ou extinguir os desafios decorrentes da implementação do BIM. }\end{array}$ \\
\hline \multicolumn{2}{|c|}{$\begin{array}{l}\text { (1) Considerou-se que a experiência e as percepções adquiridas durante a implementação do BIM pelos participantes } \\
\text { vinculados as empresas de diferentes portes (micro, pequeno, médio e grande), podem auxiliar no processo de } \\
\text { implementação do BIM em uma microempresa. Por isso, esta pesquisa valeu-se, a partir das devidas adaptações e } \\
\text { consideraçôes, das respostas de todos os participantes. } \\
\text { (2) Desafios apresentados na Tabela } 2 \text {. }\end{array}$} \\
\hline
\end{tabular}

Fonte: Autores (2021).

Finalizada a etapa "Elaboração do questionário", iniciou-se a segunda etapa "Aplicação do questionário". Para coleta das informações, o questionário de pesquisa exploratória ficou disponível no período entre 22 de julho a 3 agosto do ano de 2021, em uma plataforma de pesquisa on-line. Devido às restrições decorrentes da pandemia do COVID-19 e visando obter uma maior amostragem, o estudo foi divulgado e desenvolvido exclusivamente através da internet.

O público-alvo do questionário foram profissionais e empresas notadamente do setor AEC. A amostragem foi estabelecida conforme disponibilidade, acessibilidade, tempo e recursos, porém, visou-se a abrangência nacional na aplicação do questionário. Os profissionais participaram de forma anônima e voluntária da pesquisa e foram informados dos objetivos e benefícios da pesquisa, assim como da finalidade acadêmica e do caráter público dos resultados obtidos.

Na terceira etapa "Tratamento dos dados" foi realizado, segundo os métodos qualitativo e quantitativo, o tratamento dos dados coletados na etapa anterior, filtrando, organizando e esquematizando as informações necessárias à quarta etapa.

O meio definido para apresentação dos resultados foi a utilização de gráficos e tabelas elaborados a partir de uma análise de conteúdo sobre as respostas obtidas. A análise dos dados qualitativos foi descrita através de distribuição de frequências simples (n) e relativa (\%) que permitiram a criação de gráficos e quadros que ordenam e classificam as informações obtidas através do questionário.

Por fim, a quarta etapa consistiu na análise das informações coletadas, buscando extrair informações e produzir recursos para auxiliar no processo de implementação e disseminação do BIM nas microempresas.

\section{Resultados e Discussão}

\subsection{Resultados e análise descritiva}

A população amostral desta pesquisa foi de 47 participantes com abrangência nos dez estados do Brasil, Minas Gerais (MG), São Paulo (SP), Paraná (PR), Distrito Federal (DF), Santa Catarina (SC), Bahia (BA), Goiás (GO), Rio de Janeiro (RJ) e Rio Grande do Sul (RS), cuja maior participação foi na região Sudeste, seguida da região Sul, conforme ilustrado na Figura 1. 
Figura 1. Unidades federativas alcançadas pela pesquisa.

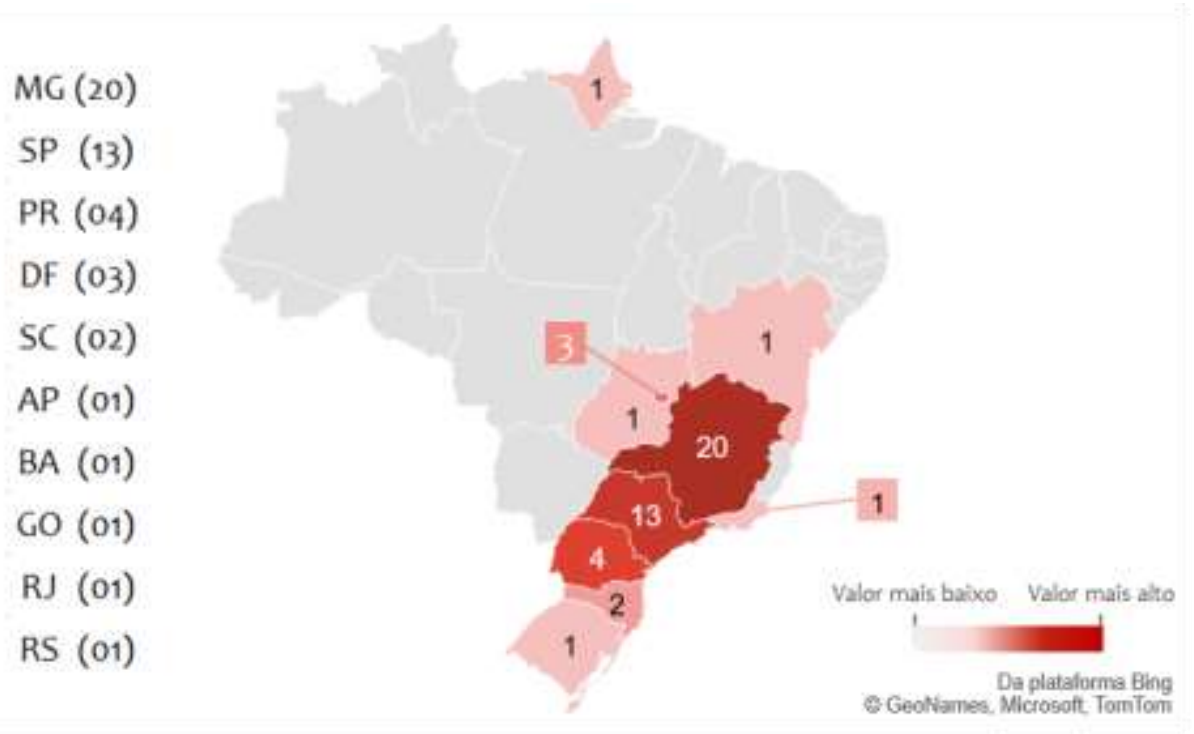

Fonte: Autores (2021).

O resultado descritivo da pesquisa é apresentado na Tabela 3.

Tabela 3. Percepção do participante quanto à aplicabilidade e nível de dificuldade dos desafios.

\begin{tabular}{|c|c|c|c|}
\hline Informação & Estratificação & $\mathbf{n}$ & $\%$ \\
\hline \multirow{2}{*}{ Sabe a definição de BIM } & Sim & 47 & 100 \\
\hline & Não & 0 & 0 \\
\hline \multirow{6}{*}{ Faixa etária (anos) } & $<18$ & 0 & 0 \\
\hline & $18-27$ & 13 & 28 \\
\hline & $28-37$ & 20 & 43 \\
\hline & $38-47$ & 8 & 17 \\
\hline & $48-57$ & 6 & 13 \\
\hline & $\geq 58$ & 0 & 0 \\
\hline \multirow{4}{*}{ Porte da empresa ${ }^{(1)}$} & Microempresa & 14 & 30 \\
\hline & Pequeno porte & 13 & 28 \\
\hline & Médio porte & 4 & 9 \\
\hline & Grande porte & 16 & 34 \\
\hline \multirow{9}{*}{ Setor de atuação ${ }^{(2)}$} & Engenharia & 31 & - \\
\hline & Arquitetura & 21 & - \\
\hline & Consultoria & 17 & - \\
\hline & Construção & 16 & - \\
\hline & Educação & 1 & - \\
\hline & Governo & 1 & - \\
\hline & Incorporação & 1 & - \\
\hline & Siderurgia & 1 & - \\
\hline & Tecnologia & 1 & - \\
\hline \multirow{2}{*}{ Conhecimento de algum instrumento facilitador da implementação do BIM } & Sim & 44 & 94 \\
\hline & Não & 3 & 6 \\
\hline \multirow{2}{*}{ Conhecimento de norma técnica brasileira referente a implementação do BIM } & Sim & 40 & 85 \\
\hline & Não & 7 & 15 \\
\hline
\end{tabular}

(1) Microempresas (Até nove colaboradores); Empresas de pequeno porte (De 10 a 49 colaboradores); Empresas de médio porte (De 50 a 99 colaboradores); Empresa de grande porte (100 ou mais colaboradores) (SEBRAE, 2020).

(2) Autodeclaração, com possibilidade de múltipla escolha. 
Observou-se na Tabela 3, a partir da faixa etária dos participantes, que a pesquisa não alcançou profissionais sêniores (58 anos ou mais). Uma possível justificativa é o fato dos indivíduos dessa faixa etária ainda estarem pouco integrados às redes sociais e plataformas digitais, mesmo naquelas de caráter profissional, conforme destacado por Hootsuite (2021).

Verificou-se que todos os participantes sabem a definição do BIM e a maioria dos participantes indicou ter conhecimento sobre os instrumentos facilitadores e normas técnicas brasileiras relacionadas ao BIM. Nesse sentido, cabe destacar que as ações de divulgação do BIM aos profissionais do setor, podem estar sendo efetivas.

Notou-se que dentre os participantes, embora a pesquisa tenha abrangido profissionais de diferentes setores de atuação, aqueles atuantes em empresas de engenharia, arquitetura, consultoria e construção foram, nessa ordem, os que apresentaram maior representatividade nesta pesquisa.

Na Figura 2 é apresentado a relação entre o porte da empresa a qual o participante está vinculado e o status da implementação do BIM nesta empresa. O status pode assumir cinco posições: (1) Não implementou o BIM e não tem intenção de implementar; (2) Não implementou o BIM, mas pretende implementar; (3) Está iniciando a implementação do BIM; (4) Está finalizando a implementação do BIM; e (5) Já implementou o BIM.

Figura 2. Status de implementação do BIM conforme os portes da empresa.

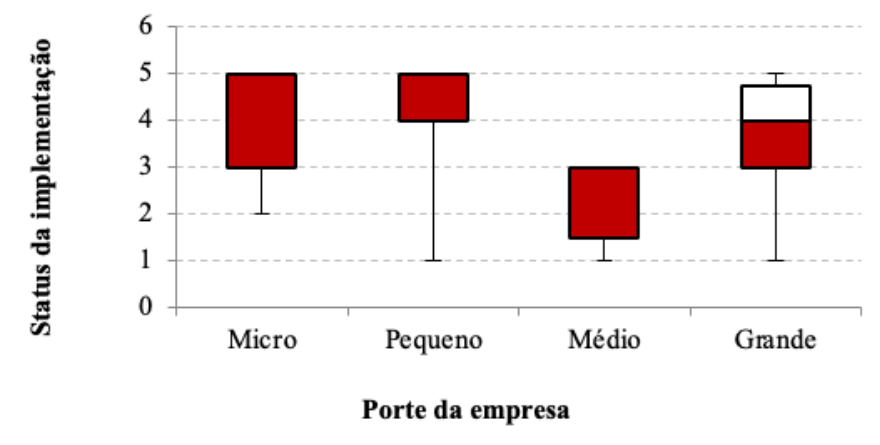

Fonte: Autores (2021).

Observa-se na Figura 2, a partir da medida do status, mediana (5), que as micro e pequenas empresas já iniciaram e/ou implementaram o BIM. Enquanto, as empresas de médio porte estão iniciando a implementação do BIM (3) e as de grande porte estão finalizando o processo de implementação do BIM (4). Uma possível justificativa para essa diferença está relacionada a dificuldade, por partes das médias e grandes empresas na mudança do processo de projeto, visto que, devido ao seu porte, tendem a necessitar de mais tempo para a implementação e realização de ajustes em processos já consolidados na empresa.

Nota-se que apenas a microempresa não teve representatividade no status de valor (1), indicando uma ampla intenção em migrar para o BIM. Deste modo, infere-se que a maioria dos participantes, representando suas respectivas empresas, entenderam a importância do BIM e possuem a intenção de acompanhar a tendência do setor, consequentemente, aproveitando oportunidades de mercado.

\subsection{Desafios na implementação do BIM em microempresas}

A partir das avaliações realizadas pelos participantes, a respeito da aplicabilidade dos desafios e da percepção pessoal do nível de dificuldade gerados por estes ao processo de implementação do BIM nas microempresas do setor AEC, foi elaborado o Quadro 1, em que é apresentado a classificação dos desafios (por ordem de dificuldade e frequência).

O quadro mostra a classificação dos em alto, médio ou baixo nível de dificuldade a partir da representação por cores, 
sendo elas vermelho, amarelo e verde, respectivamente, e aqueles que não apresentam nenhum nível de dificuldade é representado pela cor roxa.

Quadro 1. Percepções dos participantes quanto à aplicabilidade e nível de dificuldade dos desafios.

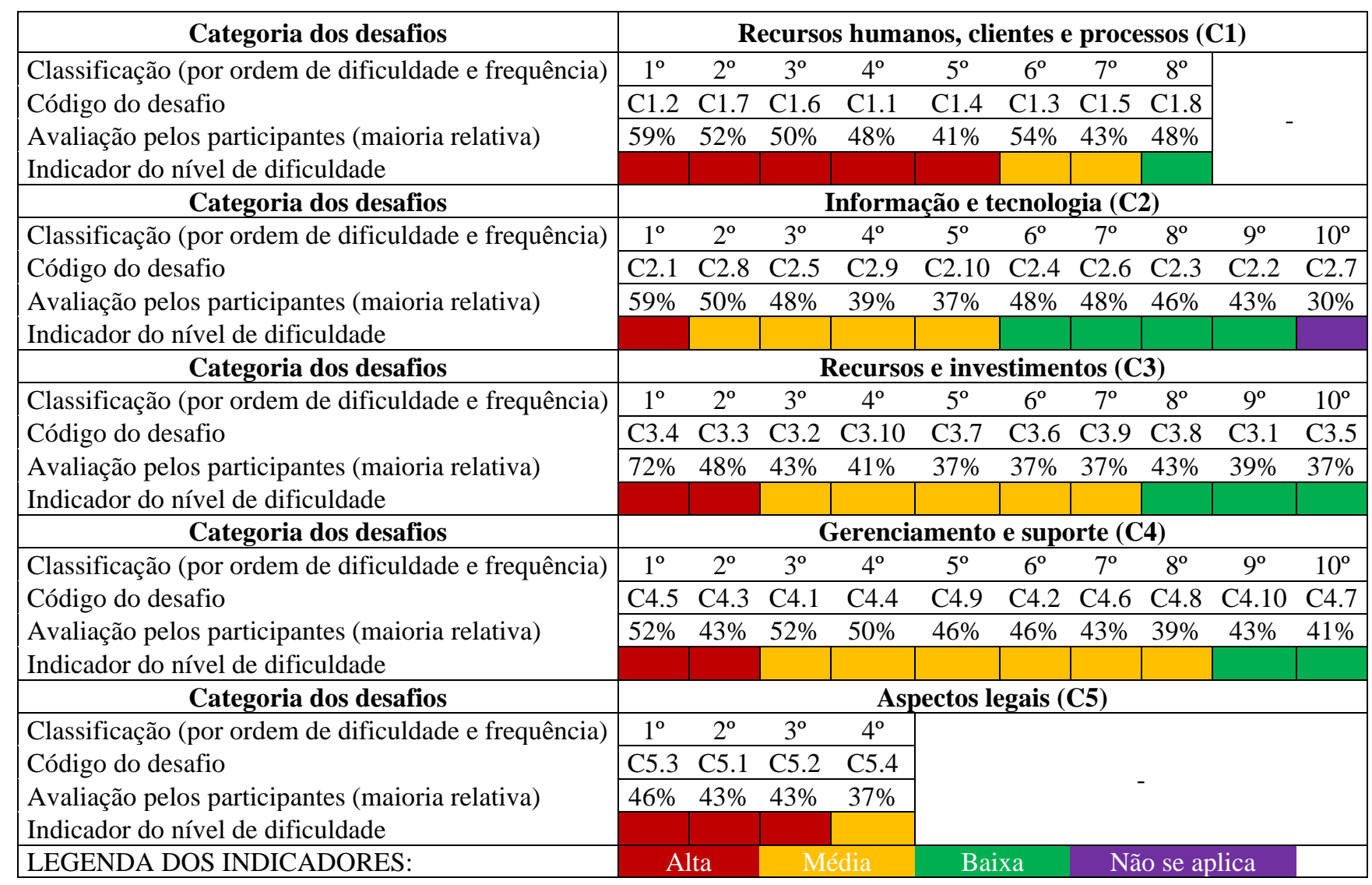

Fonte: Autores (2021).

A partir das informações organizadas no Quadro 1 é possível observar que apenas o desafio C2.7 "Falta de um software único para todas as disciplinas de projeto" não é um desafio aplicável ao processo de implementação do BIM independente do porte da empresa. Isso pode indicar um bom nível de entendimento dos participantes sobre um conceito fundamental do BIM, visto que a "Existência de um único software" seria algo contrário à integração e interoperabilidade proposta pelo BIM.

Dentre os desafios indicados com alto nível de dificuldade, houve destaque para o desafio C3.4 "Custo do investimento na aquisição e melhorias de infraestrutura tecnológica (hardwares, softwares, licenças e bibliotecas BIM)", com indicação dos 72\% participantes. Seguido por um empate entre C1.2 "Desconhecimento, por parte dos clientes, sobre as vantagens do BIM" (59\%) e C2.1 "Deficiência no ensino técnico e universitário brasileiro sobre o BIM" (59\%). Ainda em relação ao ranqueamento tem-se o desafio C4.5 "Dificuldade no gerenciamento da mudança cultural", onde 42\% dos participantes indicar como um desafio de alta dificuldade.

Ao solicitar que os participantes ordenassem as categorias em função dos desafios que a compõem e relacionando com as informações presentes no Quadro 1, foi possível observar que, apesar do desafio mais impactante (C3.4) pertencer à categoria 'Recursos e Investimentos', 43\% dos participantes indicaram a categoria de 'Recursos humanos, Clientes e Processos' como a detentora dos desafios mais relevantes. Uma possível justificativa é a maior quantidade de desafios com alto nível dificuldade (5) pertencentes a 'Recursos humanos, Clientes e Processos'. Este fato era esperado, pois, a mudança de 
paradigma tende a afetar diretamente as pessoas e processos da empresa, como destacado por CBIC (2016b).

Além de validar os desafios presentes na literatura analisando sua aplicabilidade à realidade das microempresas, foram obtidos através da participação dos profissionais 13 desafios adicionais aos encontrados na literatura, conforme elencados na Tabela 4.

Destacam se entre os desafios adicionais, uma maior quantidade de desafios relacionados a categoria 'Recursos humanos, Clientes e Processos' e ainda questões relacionadas ao custo da transição para o BIM que, por ser alto, pode tornar a implementação impraticável para algumas microempresas, reforçando o impacto que esses parâmetros possuem no processo de implementação do BIM.

Tabela 4. Desafios citados pelos participantes.

\begin{tabular}{|c|c|c|c|}
\hline Categoria & Código & $\begin{array}{c}\text { Amostra } \\
(\mathbf{n})\end{array}$ & Desafio \\
\hline \multirow{6}{*}{ 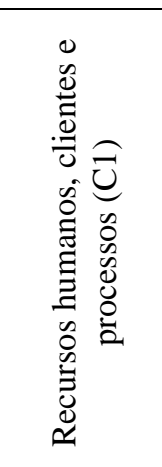 } & $\mathrm{C} 1.1+$ & 1 & $\begin{array}{l}\text { A crença que o BIM é o fim e não o meio pelo qual evoluiremos os processos de projeto, } \\
\text { construção e operação. }\end{array}$ \\
\hline & $\mathrm{C} 1.2+$ & 1 & $\begin{array}{l}\text { Falta compreensão do cliente sobre o que ele recebe como produto ao contratar projetos } \\
\text { BIM. }\end{array}$ \\
\hline & $\mathrm{C} 1.3+$ & 1 & Dificuldades com profissionais de liderança que não entendem do processo. \\
\hline & C1.4+ & 1 & $\begin{array}{l}\text { Dificuldade em convencer o investidor a adotar o BIM com foco na Gestão de Ativos } \\
\text { (Facilities Management). }\end{array}$ \\
\hline & C1.5+ & 1 & $\begin{array}{l}\text { Dificuldade quanto a definição clara e antecipada do Fluxo das Informações, envolvendo } \\
\text { todos os stakeholders de um projeto. }\end{array}$ \\
\hline & $\mathrm{C} 1.6+$ & 1 & $\begin{array}{l}\text { Dificuldade devido ao mercado privado local não reconhecer e pagar por projetos mais } \\
\text { elaborados, como os feitos em BIM. }\end{array}$ \\
\hline \multirow{3}{*}{ 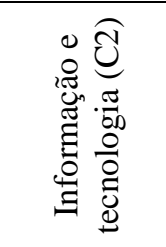 } & $\mathrm{C} 2.1+$ & 1 & $\begin{array}{l}\text { Dificuldade devido a menor flexibilidade do BIM no desenvolvimento de projetos simples, } \\
\text { quando comparado a liberdade do CAD. }\end{array}$ \\
\hline & $\mathrm{C} 2.2+$ & 1 & Dificuldade da ascensão plena do BIM no cenário empresarial. \\
\hline & $\mathrm{C} 2.3+$ & 1 & $\begin{array}{l}\text { Dificuldade em determinar o nível de detalhamento desejada para um projeto e necessário } \\
\text { para todo o seu ciclo de vida. }\end{array}$ \\
\hline \multirow{2}{*}{ 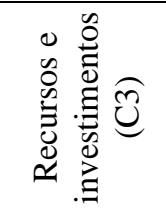 } & $\mathrm{C} 3.1+$ & 1 & Dificuldade quanto ao custo operacional de uma microempresa. \\
\hline & $\mathrm{C} 3.2+$ & 2 & $\begin{array}{l}\text { Dificuldade devido ao alto custo envolvido na transição para o BIM, que é muitas vezes } \\
\text { impraticável para empresas de menor porte. }\end{array}$ \\
\hline \multirow{2}{*}{ 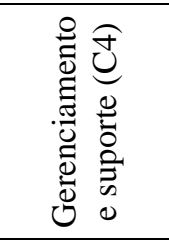 } & $\mathrm{C} 4.1+$ & 1 & $\begin{array}{l}\text { Dificuldade em conciliar o BIM com o Sistema de Gestão da Qualidade, que já tem seus } \\
\text { próprios mecanismos de controle. }\end{array}$ \\
\hline & C4.2+ & 1 & $\begin{array}{l}\text { Dificuldade inicial em perceber os benefícios do BIM de uma maneira cíclica, isto é, do } \\
\text { escritório para o canteiro de obras e durante a utilização do produto final. }\end{array}$ \\
\hline
\end{tabular}

Fonte: Autores (2021).

As opiniões dos participantes, convergem para a necessidade de maiores incentivos fiscais e financeiros, ações de iniciativas e políticas normalização e iniciativas para capacitação, discussão e disseminação da tecnologia.

\section{Considerações Finais}

O estudo identificou os desafios relacionados a implementação do BIM nas microempresas, o que auxilia no processo de implementação do BIM nas microempresas do setor AEC através da proposição de estratégias específicas para os desafios identificados.

Observou-se que no processo de implementação do BIM nas microempresas existem diversos desafios com diferentes 
níveis de dificuldade, tendo sido identificados 54 desafios aplicáveis a realidade das empresas desse porte. Além disso, definiuse que os desafios podem ser categorizados em: (i) Recursos humanos, clientes e processos; (ii) Informação e tecnologia; (iii) Recursos e investimentos, (iv) Gerenciamento e suporte; e (v) Aspectos legais.

A maior dificuldade gerada pelos desafios da implementação relacionou-se com o investimento demandado para capacitação dos colaboradores e para adequação da infraestrutura tecnológica de TI (Tecnologia da Informação) da empresa. Além disso, destacam-se os efeitos negativos da desinformação acerca da tecnologia e da deficiência no ensino técnico e universitário sobre o BIM. Apesar de importantes, apresentaram-se menos significantes, quando comparado com os demais desafios, aqueles relacionados ao retorno do investimento no curto prazo e precificação dos projetos em BIM.

A determinação, pelos participantes, de que os 41 desafios identificados na literatura, são aplicáveis a realidade das microempresas, é de suma importância para proposição de estratégias na implementação. E esses desafios podem impactar positivamente na elaboração do planejamento da implementação do BIM, uma vez que foram organizados e categorizados, promovendo a otimização do tempo em relação tratamento dessas informações.

Adicionalmente, mostra-se interessante, como um desdobramento da pesquisa, uma revisão literária e uma pesquisa de campo, acerca de estratégias para a mitigação dos desafios presentes na implementação do BIM em microempresas.

\section{Referências}

Agência Brasileira de Desenvolvimento Indústrial. ABDI. (2017) GUIA 6 - A Implantação de Processos BIM. Coletânea de Guias BIM ABDI-MDIC.

Brasil (2017). Decreto nº 9.377, de 17 de maio de 2017. Brasília: Presidência da República.

Brasil (2019). Decreto n 9.983, de 22 de agosto de 2019. Brasília: Presidência da República

Brasil (2020). Decreto nº 10.306, de 2 de abril de 2020. Brasília: Presidência da República.

Brasil (2021). Lei n ${ }^{\circ} 14.133$, de $1^{\text {o }}$ de abril de 2021. Brasília: Presidência da República.

Brasil (2018). Ministério da Economia. Governo federal lança estratégia para promover inovação na indústria da construção. Ministério da Economia, Brasília.

Campestrini, T. F., Garrido, C. G., Mendes Jr., R., Scheer, S, Freitas, M. C. D. (2015) Entendendo BIM. G 2015 Entendendo BIM.

Câmara Brasileira da Indústria da Construção, CBIC. (2016a). Fundamentos BIM - Parte 1: Fundamentos BIM, Coletânea Implementação do BIM para Construtoras e Incorporadoras. v.1. Brasília. 124p.:il.

Câmara Brasileira da Indústria da Construção, CBIC. (2016b) Fundamentos BIM - Parte 2: Implementação BIM, Coletânea Implementação do BIM para Construtoras e Incorporadoras. v.2. Brasília. 72p.:il.

Câmara Brasileira da Indústria da Construção, CBIC. (2016c). 10 motivos para evoluir com o BIM. Brasília.

Câmara Brasileira da Indústria da Construção, CBIC. (2018). Road Show BIM: Resultados da pesquisa e desdobramentos. Brasília.

Cardoso, R. R., Souza, E. M., Matta, P. S., Santos, F. R. (2020). Aspectos contrutivos no uso do BIM. Episteme Transversalis, 11 (3), $264-277$.

Carvalho, J. R. (2019). Vantagens do sistema BIM nos ambientes de projeto e gestão da construção civil. (Monografia) Universidade de Taubaté.

Carvalho, G. D. G. de, Silva, W. da V., Santos, P., A. C., Gomes, H. C. (2015). Radar da inovação como ferramenta para o alcance de vantagem competitiva para micro e pequenas empresas. RAI Revista de Administração e Inovação, 12(4), 162-186.

Castro, B. de (2020). BIM, a inteligência da construção. ABDI.

Chan, D. W. M., Olawumi, T. O., Ho, A. M. L. (2019). Perceived benefits of and barriers to Building Information Modelling (BIM) implementation in construction: The case of Hong Kong. Journal of Building Engineering, v. 25.

Checcucci, E. de S. (2019). Teses e dissertações brasileiras sobre BIM: uma análise do período de 2013 a 2018. PARC Pesquisa em Arquitetura e Construção, 10 (1), https://doi.org/10.20396/parc.v10i0.8653708.

Coelho, K. M. (2017). A implementação e o uso da modelagem da informação da construção em empresas de projeto de arquitetura. (Tese de Doutorado). Universidade de São Paulo.

Coutinho, A. B., Moura, G. S., Teixeira, E. K. C. (2021). Compatibilização de um projeto arquitetônico e hidrossanitário utilizando a metodologia BIM. Research, Society and Development, 10(2), 1-13. 
Costa, G. C. L. R., Figueiredo, S. H., Ribeiro, S. E. C. (2015). Estudo comparativo da tecnologia CAD com a tecnologia BIM. Revista de Ensino de Engenharia, 34(2).

Dantas Filho, J. B. P., Borges, A. V. G., Soares, G. N., Souza, D. S. V., Guerra, R. S., Cardoso, D. R., Barros Neto, J. P. (2015). Estado de adoção do Building Information Modeling (BIM) em empresas de arquitetura, engenharia e construção de Fortaleza/CE. In: Encontro brasileiro de tecnologia de informação e comunicação na construção, 7., 2015, Recife. Anais [...] Porto Alegre: ANTAC, 20151-7.

DataSebrae. (2020). Painel de Empresas 2020.

Eastman, C., Teicholz, P., Sacks, R., Liston, K. (2021). Manual de BIM: Um Guia de Modelagem da Informação da Construção para Arquitetos, Engenheiros, Gerentes, Construtores e Incorporadores. Bookman Editora.

Ghaffarianhoseini, A, Tookey, J., Ghaffarianhoseini, A., Naismith, N., Azhar, S., Efimova, O., Raahemifar, K. (2017) Building Information Modelling (BIM) uptake: Clear benefits, understanding its implementation, risks and challenges. Renewable and Sustainable Energy Reviews, 75, 1046-1053.

Gomes, R.M.S., Abreu, A.A., Bauer, M.A., Nascimento, G.C., Vale, M.S. (2018) Modelagem de informações para a construção (BIM): ambientes colaborativos para gestão de projetos e obras na construção civil. Perspectivas On-line: Exatas \& Engenharia, 8 (23), 48 - 62.

Governo do Paraná. (2018). Caderno de especificações técnicas para contratação de projetos em BIM - Edificações. Caderno BIM. Coletânea cadernos orientadores Secretaria de Estado de Infraestrutura e Logística ,Curitiba.

Hootsuite, We are social. (2021). Digital 2021: Brazil.

Lakatos, E. M.; Marconi, M. de A. (2017). Fundamentos de metodologia científica. 8. ed. São Paulo: Atlas.

Landim, A. F. G. (2020). Os obstáculos à implantação da tecnologia BIM como plataforma no desenvolvimento de projetos na construção civil: uma revisão sistemática de literatura. (Monografia). Instituto Federal de Educação, Ciência e Tecnologia Paraíba.

Liu, S., Xie, B., Tivendal, L. Liu, C. (2015). Critical barriers to BIM implementation in the AEC industry. International Journal of Marketing Studies, 7(6), $162-171$.

Maína, J. (2018). CAD and BIM in architecture education: awareness, proficiency and advantages from the student perspective. Gazi University Journal of Science Part B: Art Humanities Design and Planning, 6 (4), 167-178

Migilinskas, D., Vladimir, P., Juocevicius, V., Ustinovichius, L. (2013). The benefits, obstacles and problems of practical BIM implementation. Procedia Engineering, 57, 767-774.

Pereira, M. D. dos S. (2016). Implementação do BIM nas organizações: práticas e sugestões para a implementação. (Tese de Doutorado) Universidade do Minho.

Santos, A. C. V. (2016) Implantação do sistema BIM no ato de projetar do Departamento de Projetos da UFMG. (Monografia). Universidade Federal de Minas Gerais.

Santos, D. V., Lima, M. M. X., Campos, V. R. (2016). Análise da percepção do uso do BIM em diferentes implantações: estudo de caso. In: Encontro Nacional de Tecnologia do Ambiente Construído, São Paulo. Anais [...]. São Paulo: ANTAC.

Serviço brasileiro de apoio às micro e pequenas empresas. Sebrae. (2020). Atualização de Estudo sobre a Participação de Micro e Pequenas Empresas na Economia Nacional. Unidade de Gestão Estratégica.

Sienge e Grant Thornton. (2020). Mapeamento de maturidade BIM Brasil.

Silva, G. \& Dacorso, A. L. R. (2014). Riscos e incertezas na decisão de inovar das micro e pequenas empresas. RAM. Revista de Administração Mackenzie, 15 (4), 229-255.

Smith, P. (2014). BIM Implementation. Global Strategies, Procedia Engineering, 85 (1), 482-492. https://doi.org/10.1016/j.proeng.2014.10.575

Sun, C., Jiang, S., Skibniewski, M. J., Qingpeng, H., Shen, L. (2017). A literature review of the factors limiting the application of BIM in the construction industry. Technological and Economic Development of Economy, 23 (5), 764-779.

Steiner, L. R. (2016) Análise da implementação da plataforma BIM no setor da AEC do Estado de Santa Catarina. (Monografia) Unisul.

Stehling, M. P. \& Arantes, E. M. (2014) Análise do processo de implantação de BIM em empresas de projetos industriais e arquitetônicos em Belo Horizonte. Pesquisa em Arquitetura e Construção, 5 (1), 35-44.

Succar, B. \& Kassem, M. (2016). Building Information Modelling: Point of adoption. In: CIB World Conference Proceedings.

Zeglin, B. V., Reis, A. M., Vergara, L. G. L. (2018) Challenges of implementing Building Information Modeling (BIM) in the Construction Industry. XXII Congresso da Sociedade Iberoamericana de Gráfica Digital. 